\title{
Revista Colombiana de

\section{Infrautilización de la anticoagulación oral en el paciente octogenario con fibrilación auricular: perspectiva desde la práctica clínica real}

\section{Verónica Hernández ${ }^{\mathrm{a}, *}$, Jesús Saavedra ${ }^{\mathrm{b}}$, Virginia Mazoteras ${ }^{\mathrm{c}}$ y María T. López ${ }^{\mathrm{d}}$}

\author{
a Servicio de Cardiología, Hospital General de Ciudad Real, Ciudad Real, España \\ b Servicio de Cardiología, Hospital Universitario de Getafe, Getafe, Madrid, España \\ c Servicio de Geriatría, Hospital General de Ciudad Real, Ciudad Real, España \\ ' Servicio de Cardiología, Hospital General de Ciudad Real, Ciudad Real, España
}

Recibido el 24 de noviembre de 2014; aceptado el 3 de junio de 2015

Disponible en Internet el 15 de julio de 2015

\section{PALABRAS CLAVE}

Fibrilación auricular; Embolización; Hemorragia; Anticoagulación

\begin{abstract}
Resumen
Introducción y objetivos: La fibrilación auricular es la arritmia más frecuente en los pacientes ancianos. La decisión de la anticoagulación en este grupo puede ser difícil. Quisimos estudiar el manejo de la anticoagulación oral en pacientes muy mayores con fibrilación auricular en la práctica clínica real y los factores asociados con la decisión de anticoagular.

Métodos: Estudiamos todos los pacientes mayores de 80 años de edad, con fibrilación auricular conocida atendidos en la consulta de geriatría de junio a diciembre de 2013.

Resultados: Estudiamos 90 pacientes, con edad media de 85,6 años. La media de la puntuación de CHA2DS2-VASC fue de 4,7 y la media de HAS-BLED fue de 2. El 53\% estaban anticoagulados. De los pacientes anticoagulados, el $96 \%$ tomaban acenocumarol y un $4 \%$ los nuevos anticoagulantes. En el análisis multivariante, la edad y el antecedente de demencia se asoció a la decisión de no anticoagulación.

Conclusiones: Según nuestros resultados, un alto porcentaje de pacientes muy mayores con fibrilación auricular están sin anticoagular. El antecedente de demencia y la edad pesan más que la puntuación CHAD2DS2-Vasc y HAS-BLED a la hora de decidir la anticoagulación.

(c) 2015 Sociedad Colombiana de Cardiología y Cirugía Cardiovascular. Publicado por Elsevier España, S.L.U. Este es un artículo Open Access bajo la licencia CC BY-NC-ND (http://creativecommons.org/licenses/by-nc-nd/4.0/).
\end{abstract}

\footnotetext{
* Autor para correspondencia.

Correo electrónico: veronicahernandezz@hotmail.com (V. Hernández).
} 


\section{KEYWORDS}

Atrial fibrillation; Embolization; Haemorrhage; Anticoagulation
Underuse of oral anticoagulation in octogenarian patient with atrial fibrillation: a perspective from real clinical practice

\begin{abstract}
Introduction and objectives: Atrial fibrillation is the most common arrhythmia in elderly patients. Anticoagulation decision in this group can be difficult. We wanted to study the management of oral anticoagulation in very elderly patients with atrial fibrillation in clinical practice and the factors associated with the decision to anticoagulation.

Methods: We studied all patients older than 80 years with known atrial fibrillation treated at the geriatric consultation from June to December 2013.

Results: We studied 90 patients with a mean age of 85.6 years. The mean score of CHA2DS2VASc was 4.7 and the mean of HAS-BLED was $2.53 \%$ were anticoagulated. Out of anticoagulated patients, $96 \%$ were taking Acenocumarol and $4 \%$ new anticoagulants. In multivariate analysis, age and history of dementia was associated with the decision not to use anticoagulants.

Conclusions: Our results suggest that a high percentage of very elderly patients with atrial fibrillation are without anticoagulation. The history of dementia and age outweigh the CHAD2DS2-Vasc and HAS-BLED score on deciding to anticoagulate.

(C) 2015 Sociedad Colombiana de Cardiología y Cirugía Cardiovascular. Published by Elsevier España, S.L.U. This is an open access article under the CC BY-NC-ND license (http://creativecommons.org/licenses/by-nc-nd/4.0/).
\end{abstract}

\section{Introducción}

La fibrilación auricular (FA) es la arritmia más frecuente en los pacientes ancianos, y su prevalencia aumenta con la edad, afectando al $5 \%$ de los pacientes mayores de 65 años de edad, y al $10 \%$ de los mayores de 80 años $^{1}$. La población anciana tiene un alto riesgo de ictus y de embolias sistémicas por lo que estaría indicada en la mayoría de ellos la anticoagulación oral ${ }^{2}$. No obstante, la anticoagulación oral está infrautilizada en este grupo etario. Según algunos estudios hasta un $50 \%$ de los pacientes con indicación de anticoagulación no la reciben. Las principales razones para no prescribir anticoagulación en estos pacientes son: el alto riesgo de sangrado, la comorbilidad asociada, la polifarmacia y el elevado riesgo de caídas ${ }^{3}$. Las guías clínicas actuales consideran la edad como un factor de riesgo tanto de ictus como de sangrado lo que puede generar incertidumbre. Además, los pacientes octogenarios no están incluidos en la mayoría de los ensayos clínicos aleatorizados ${ }^{4}$.

De acuerdo con esto, quisimos estudiar la utilización en un entorno clínico real de la anticoagulación oral en pacientes de la tercera edad con FA ya conocida, atendidos en la consulta de geriatría y analizar qué factores influían en la no prescripción de la anticoagulación.

\section{Métodos}

Estudiamos de forma retrospectiva todos los pacientes mayores de 80 años de edad con FA ya conocida atendidos en la consulta de geriatría desde junio de 2013 a diciembre del mismo año. Recogimos datos epidemiológicos, clínicos y analíticos así como el tratamiento que recibían (tabla 1). Valoramos el estado funcional de los pacientes con la escala Barthel que va de 0 a 100 (puntuación 100: capacidad funcional normal $)^{5}$. Calculamos el riesgo embólico según la escala
$\mathrm{CHA}_{2} \mathrm{DS}_{2}-\mathrm{VASC}^{6}$ y el riesgo hemorrágico según la escala HAS$\mathrm{BLED}^{7}$.

\section{Análisis estadístico}

Para el análisis de datos se utilizó el paquete estadístico Stata versión 12.1 (Stata Corp, 4905 Lake Way, Texas, Estados Unidos). Las variables continuas se expresaron como media y desviación estándar (DS) y las variables categóricas en valor absoluto y porcentaje. Para comparar las

\section{Tabla 1 Características de los pacientes}

\begin{tabular}{ll}
\hline Edad (media $\pm D E)$ & $85,6 \pm 4,5$ \\
Sexo femenino (\%) & 59 \\
Modo de vida (\%): & 6 \\
Solo & 86 \\
Con un familiar & 8 \\
Institucionalizado & $63,1 \pm 3,3$ \\
Barthel (media $\pm D E$ ) & \\
Comorbilidad asociada (\%): & 95,6 \\
Hipertensión & 31,1 \\
Diabetes mellitus & 25,6 \\
Patología pulmonar & 52,2 \\
Insuficiencia cardiaca previa & 21,1 \\
C. isquémica crónica & 25,6 \\
Demencia & 16 \\
Ictus previo & \\
Patrón de FA (\%): & 21 \\
Paroxística & 3 \\
Persistente & 66 \\
Permanente & $4,7 \pm 1,2$ \\
CHADS-VASC (media $\pm D E)$ & $2 \pm 0,7$ \\
HAS-BLED (media $\pm D E$ ) &
\end{tabular}

DE: desviación estándar; FA: fibrilación auricular. 
Tabla 2 Análisis univariante y multivariante de los factores asociados a la decisión de no anticoagulación

\begin{tabular}{lcc}
\hline & Univariante (odds ratio, IC al 95\%; $p$ ) & Multivariante (odds ratio, IC al 95\%; $p$ ) \\
\hline Edad & 0,$9 ; \mathrm{IC}: 0,81-0,95 ; p=0,04$ & $0,89, \mathrm{IC}: 0,8-0,98 ; p=0,03$ \\
Demencia & $0,27, \mathrm{IC}: 0,1-0,77 ; p=0,01$ & $0,25, \mathrm{IC}: 0,08-0,73 ; p=0,01$ \\
Antecedente de insuficiencia cardiaca & $2,45, \mathrm{IC}: 1,05-5,71 ; p=0,04$ & $2,3, \mathrm{IC}: 0,93-5,81 ; p=0,06$ \\
\hline
\end{tabular}

IC: intervalo de confianza.

proporciones se utilizó la prueba de Chi Cuadrado $\left(\chi^{2}\right)$ y para comparar variables cuantitativas se utilizó la t de Student. Se comprobó el supuesto de normalidad con el test de Shapiro-Wilk. Se realizó un análisis univariante y multivariante mediante regresión logística para identificar las variables asociadas a la decisión de anticoagular. Para todas las pruebas estadísticas se consideró un resultado estadísticamente significativo un valor $\mathrm{p}<0,05$ y se calcularon los intervalos de confianza al $95 \%$.

\section{Resultados}

Se estudiaron un total de 90 pacientes con una media de edad de 85,6 años (DE 4,5). Las características de los pacientes quedan recogidas en la tabla 1 . La media de la puntuación de la escala $\mathrm{CHA}_{2} \mathrm{DS}_{2}$-VASC fue de 4,7 (DE 1,2) y la media de la escala HAS-BLED fue de 2 (DE 0,7). El 53\% de los pacientes estaban anticoagulados, de ellos el $96 \%$ recibían acenocumorol y el $4 \%$ los nuevos anticoagulantes. El 94\% de los sujetos no anticoagulados recibían algún antiagregante.

En el análisis univariante no hubo diferencias estadísticamente significativas en la prescripción de la anticoagulación según el patrón de FA, el modo de vida de los pacientes, el sexo, la presencia de déficit visual o auditivo ni del estado funcional valorado por la escala Barthel. No encontramos diferencias estadísticamente significativas en la puntuación de las escalas de $\mathrm{CHA}_{2} \mathrm{DS}_{2}$-VASC y HAS-BLED entre los pacientes anticoagulados y los no anticoagulados.

Encontramos que los factores que sí se asociaban con la decisión de no anticoagulación eran una edad más avanzada, el antecedente de demencia y no tener antecedente de insuficiencia cardiaca. En el análisis multivariante, solo la edad y el antecedente de demencia se asociaron de forma significativa con la decisión de no anticoagulación (tabla 2).

\section{Discusión}

Los pacientes ancianos con FA, tienen un riesgo elevado de ictus ya que solamente considerando la edad, la puntuación en la escala $\mathrm{CHA}_{2} \mathrm{DS}_{2}$-VASC es de dos y de acuerdo con las guías clínicas actuales deberían estar anticoagulados ${ }^{6}$. No obstante, la edad también es un factor de riesgo de sangrado por lo que la decisión de anticoagular a un paciente anciano puede resultar complicada para el médico. Los datos disponibles sobre la anticoagulación oral en los ancianos son limitados y este tipo de pacientes no suelen estar incluidos en los ensayos clínicos.

En nuestro estudio, los pacientes a pesar de tener un alto riesgo embólico y un riesgo moderado de hemorragia solamente el $53 \%$ de los casos estaban anticoagulados. Este porcentaje es similar a otros trabajos ya publicados ${ }^{1}$. En el trabajo publicado por Lotze et al. en el que se incluyeron pacientes ingresados de $\geq 80$ años con FA, solamente el $26 \%$ fueron dados de alta con anticoagulantes orales ${ }^{8}$. En el estudio de Denoël et al. en el que se incluyeron pacientes con FA algo más jóvenes ( $\geq 75$ años), el $61 \%$ de ellos recibieron anticoagulación oral ${ }^{9}$.

Observamos, además, la baja proporción en la prescripción que suponen los nuevos anticoagulantes orales. Estos nuevos fármacos han demostrado no ser inferiores en la prevención del ictus respecto a los antagonistas de la vitamina $\mathrm{K}$, con un riesgo de sangrado similar o menor ${ }^{10}$, evitando las fluctuaciones del INR y la necesidad de una estrecha monitorización de INR por lo que varios ensayos clínicos consideran que los nuevos anticoagulantes deben ser considerados en los pacientes ancianos con indicación de anticoagulación ${ }^{11}$.

Comprobamos que en la práctica diaria real, en la decisión de anticoagular al paciente anciano, valoramos más otros factores como la edad y el antecedente de la demencia que hacen referencia a la fragilidad, al estado funcional y al mental y se tiene menos en cuenta la puntuación de la escala $\mathrm{CHA}_{2} \mathrm{DS}_{2}$-VASC. Sería interesante añadir en las guías nuevos modelos para una mejor estratificación del riesgo de sangrado e ictus en los pacientes de edad avanzada.

\section{Responsabilidades éticas}

Protección de personas y animales. Los autores declaran que para esta investigación no se han realizado experimentos en seres humanos ni en animales.

Confidencialidad de los datos. Los autores declaran que en este artículo no aparecen datos de pacientes.

Derecho a la privacidad y consentimiento informado. Los autores declaran que en este artículo no aparecen datos de pacientes.

\section{Conflicto de intereses}

Los autores declaran no tener ningún conflicto de intereses.

\section{Bibliografía}

1. Páramo JA. Nuevos anticoagulantes orales: el dilema de la anticoagulación en el anciano. Med Clin (Barc). 2013;141(8):346-8.

2. Tulner L, Campen JV, Kuper I, Gijsen G, Koks C, Gillavry M, et al. Reasons for undertreatment with oral anticoagulants in frail geriatric outpatients with atrial fibrillation. Drugs Aging. 2010 Jan;27(1):39-50.

3. Denoël P, Vanderstraetn J, Mols P, Pepersack T. Could some geriatric characteriscs hinder the prescription of anticoagulants in atrial fibrillation in the elderly? J Aging Res. 2014:2014. 
4. Poli D, Antonucci E, Testa S, Tosetto A, Ageno W, Palareti G. Bleeding risk in very old patients on vitamin $\mathrm{K}$ antangonist treatment: Results of a prospective collaborative study on elderly patients followed by italian centres for anticoagulation. Circulation. 2011;124:824-9.

5. Mahoney FI, White BK. A measure of disability. Arch Environ Health. 1964;8:834-9.

6. 2014 AHA/ACC/HRS Guideline for the management of patients with atrial fibrillation: A report of the American College of Cardiology/American Heart Association Task Force on Practice Guidelines and the Heart Rhythm Society. Circulation. 2014;130:e272-4.

7. Pisters R, Lane DA, Nieuwlaat R, de Bos CB, Crijns HJ, Lip GY. A novel user-friendly score (HAS-BLED) to assess 1-year risk of major bleeding in patients with atrial fibrillation: the Euro Heart Survey. Chest. 2010;138:1093-100.
8. Lotze U, Liebetrau J, Malsch I, Fruhwirt B, Rothhagen B, Kharatyan $\mathrm{N}$, et al. Medical treatment of patients with atrial fibrillation aged over 80 years in daily clinical practice: Influence of age and $\mathrm{CHADS}_{2}$ score. Arch Gerontol Geriatr. 2010;50(1):36-41.

9. Denoël P, Vanderstraeten J, Mols P, Pepersack T. Could some geriatric characteristics the prescription of anticoagulants in atrial fibrillation in the elderly. J Aging Res. 2014;2014: $1-9$.

10. Vilchez JA, Gallego P, Lip GY. Safety of new oral anticoagulant drugs: a perspective. Ther Adv Drug Saf. 2014;5(1): $8-20$.

11. Ng KH, Hart RG, Eikelboom JW. Anticoagulation in patients aged $\geq 75$ years with atrial fibrillation: Role of novel oral anticoagulants. Cardiol Ther. 2013;2:135-49. 